\title{
Didáctica de las Ciencias Sociales y formación del profesorado
}

\section{Escenario actual de la formación de profesores de Educación Primaria}

\subsection{Punto de partida}

La razón que justifica nuestra presencia en un foro centrado en el intercambio de ideas y experiencias orientadas a generar, visualizar, movilizar, analizar, valorar, comprender y promover múltiples aspectos relacionados con los profesionales y estudiosos de una escuela "reflexiva y pensante" no es otra que nuestra actividad docente e investigadora desarrollada en el área de la Didáctica de las Ciencias Sociales en el ámbito de la formación inicial de maestros de Educación Primaria durante casi dos décadas en la Universidad de Granada. Tarea desempeñada además durante los últimos años en el contexto del proceso implantación del Espacio Europeo de Educación Superior, EEES.

En tal marco, una de las claves de nuestra personal trayectoria académica en el área de Didáctica de las CCSS consiste en el principio que define al maestro como profesional dotado de una autentica dimensión vocacional en su ejercicio laboral entendida como capacidad para elaborar el propio conocimiento y autonomía en el ejercicio de su labor. Dimensión vocacional esta que, a nuestro juicio, se ha visto amenazada a través de reiterados intentos de reducir la identidad del docente a la de mero "trabajador de la enseñanza" y la voluntad de socavar su autonomía profesional desde instancias políticas y administrativas al servicio de intereses ideológicos que han buscado "orientar" la formación de los docentes hacia "verdades establecidas" desde dictados incuestionables, carentes en bastantes casos de fundamentación e ignorantes de la legítima y real pluralidad ideológica existente tanto en la Academia como en la Sociedad.

Habiendo sido hasta hoy el campo del conocimiento social tierra abonada para fenómenos de esta índole, resulta obligado asumir el compromiso de abordar la Didáctica de las CCSS como desarrollo de un campo científico que, desde la legíitima pluralidad de paradigmas existentes, contribuya a la formación inicial de un profesorado de calidad comprometido en la mejora de la realidad docente y social. Por ello desarrollar una acción profesional en este Área de Conocimiento exige ser consciente de la complejidad de la sociedad contemporánea en todas sus dimensiones al tiempo que poseer la capacidad de percibir un entorno histórico caracterizado como realidad mutante y fragmentada, definida por algunos como "era del pensamiento débil" o tildada por otros como situación de indudable "quiebra de humanidad". Una sociedad en que ha emergido, junto a un encomiable desarrollo de los derechos y las libertades individuales y publicas, un perfil de ciudadano desbordado de información, dependiente de las nuevas tecnologías, portador de crecientes vacios interiores, limitado por llamativas dificultades de comunicación personal y,

Revista Iberoamericana de Educación / Revista Ibero-americana de Educação

ISSN: $1681-5653$

n. ${ }^{\circ} 63 / 1-15 / 09 / 13$

Organización de Estados Iberoamericanos para la Educación, la Ciencia y la Cultura (OEI-CAEU)

Organização dos Estados Ibero-americanos para a Educação, a Ciência e a Cultura (OEI-CAEU) 
sobre todo, gradualmente insatisfecho en un mundo de aparente abundancia; sobre todo en el mundo europeo y occidental.

Somos conscientes de que formar docentes provenientes de tal contexto supone un reto para el alumnado y el profesorado al tiempo que un desafio derivado del futuro de incertidumbre que nos depara la presente coyuntura histórica. Por otra parte, y desde el punto de vista académico son muchos los campos hoy sometidos a revisión en el contexto del EEES generando todo ello un ambiente de gran inestabilidad y hondo relativismo que ya ha sido descrito por numerosos teóricos sociales expertos en el fenómeno de la postmodernidad desde finales del S. XX

Conscientes de la naturaleza de nuestra actividad, resulta evidente la dificultad de articular una respuesta adecuada al conjunto de necesidades apuntadas como para el creciente número de demandas dirigidas al sistema educativo. Por ello, partimos en nuestra actividad profesional de una serie de elementos que creemos irrenunciables para la Didáctica de las CCSS; referentes sobre los que, tras un proceso de reflexión hemos articulado nuestra acción docente e investigadora en la materia Didáctica de las CCSS en el marco del programa del Grado en Maestro en Ed. Primaria. Y todo ello tras una dilatada experiencia previa desarrollada desde 1988 en prácticamente todos los niveles del sistema educativo español ${ }^{1}$ finalmente completa, tal como se ha indicado, mediante nuestra participación en el desarrollo del laborioso itinerario de establecimiento del nuevo EEES llevado a cabo en nuestra Facultad. Asi pues, y pertrechados con tal bagaje, hemos establecido como finalidad inmediata de nuestra actividad, procurar -desde la aportación que el área de la Didáctica de las CCSS pueda realizar al respecto-, la consolidación del EEES en el campo del nuevo Grado de Maestro en Educación Primaria mediante la mejora de la formación inicial de su alumnado.

\subsection{Nuevos contextos para la formación de maestros}

Tras la anterior declaración inicial de intenciones centramos ahora el discurso en una descripción más concreta de los contextos sociales emergentes que enmarcan nuestra labor formativa de las primeras generaciones de profesores de Educación Primaria del tercer milenio; realidad ésta que, más allá de su simbolismo y fenomenología de singular evento cronológico, ha significado no sólo una época de cambios sino un cambio de época. Y, ello, no sólo porque hemos estrenado una nueva etapa sino porque también se nos ha brindado la oportunidad de asistir como protagonistas, cualitativamente hablando, a un giro copernicano (Nieto 2001: 2).

En tal sentido nadie discutiría que la posmodernidad constituye un hecho universal que ha cobrado diversos matices según países o regiones hasta convertirse en un desafío cuyas características nos interpelan exigiendo una respuesta. Un reto caracterizado en primer término por el "desencanto de la

\footnotetext{
${ }^{1}$ Educación General Básica, Formación Profesional de $1 .{ }^{\circ}$ y $2 .{ }^{\circ}$ grado, Bachillerato Unificado Polivalente, Programas de Garantía Social, Educación especial y la actual Educación Secundaria Obligatoria. En el ámbito propiamente universitario, y en concreto en el área de la Didáctica de las Ciencias Sociales, contamos con la experiencia adquirida sucesivamente como Profesor Asociado, 1996; Profesor Ayudante Doctor, 2007; y Profesor Contratado Doctor, 2011 en la Facultad de Ciencias de la Educación de la Universidad de Granada impartiendo durante todo este tiempo en las anteriores especialidades de Magisterio, en la Diplomatura de Educación Social y en las Licenciaturas de Pedagogía y Psicopedagogía, asi como en los actuales nuevos Grados de Magisterio: cuatro materias troncales, una obligatoria, cinco optativas y dos de Libre Configuración. Asimismo hemos tenido oportunidad de participar en la dirección de trabajos de fin de Máster u otros de fin de ciclo, habiendo actuado como miembro de Comités evaluadores de Máster en centros universitarios españoles y extranjeros. También en el campo docente hemos desarrollado durante estos años otras actividades docentes en la Universidad de Granada concretamente en su Escuela de Posgrado, en su Centro de Formación continua, en el Aula de formación abierta y en ciertas actividades y cursos del CAP. Del mismo modo hemos impartido docencia en otros centros universitarios de México, Portugal y España
} 
razón"; fenómeno así calificado por prestarse a intereses sesgados con el fin de establecer, según sus diversas perspectivas, principios de validez universal que han generado ilusiones jamás materializadas dando lugar en no pocas ocasiones a significativos abismos entre teoría y praxis.

El desafio postmoderno viene ligado en segundo lugar al rechazo de aquellos grandes "relatos" que, a modo de teorías y narraciones han justificado abusos variados bajo pretexto de programas salvadores de la humanidad. Un rechazo que en realidad entraña un recelo frente a todo discurso omnicomprensivo que reduce la ética a algo provisional y privilegia la heterogeneidad a pesar de su riesgo de fragmentación. La tercera característica de esta posmodernidad es el "fin de la historia" pues, tras la disolución de los grandes relatos generadores de sentido, solo cabe pensar en la realización de la persona en el ámbito de la inmediatez, renunciando a toda posible utopía o atisbo de teleología. Nueva situación que parece indicar que lo esencial sólo es una actitud de búsqueda del goce hedonista inmediato acompañada de una ausencia real de compromiso y pasión.

Una "vuelta ecléctica a lo religioso" es otro de los rasgos postmodernos; sensibilidad desde la cual la religión se privatiza y mezcla, las espiritualidades se venden en el supermercado, los jóvenes vibran con figuras de prestigio sin asumir compromisos reales y la trascendencia se trivializa en horóscopos, sectas, esoterismos, experiencias místicas orientales y supersticiones. Todo lleva finalmente a la "estetización de la vida" dando lugar a un fenómeno que, como producto final, ya ha forjado casi dos generaciones de ciudadanos débiles, acríticos, desmemoriados, insensibles ante el dolor humano y representantes de una contradictoria cultura anoréxica y obesa a la vez y, finalmente, cansada.

Si bien es verdad que la posmodernidad ofrece también facetas positivas que tampoco pueden ignorarse, durante los últimos años parece haberse impuesto tal conjunto de fenómenos que ya no pasan desapercibidos para nadie en razón de sus inevitables consecuencias para el mundo educativo que es nuestra clave de interpretación de la realidad.

Tras esta posmodernidad, el segundo desafío planteado es el advenimiento de la Globalización, realidad que, con toda su carga positiva latente se concreta, entre otras, en las siguientes cuestiones (Ibídem, 3): La idea de aldea global, el descubrimiento de que somos una cultura planetaria que tiende a superar fronteras y barreras; la apertura al diálogo intercultural y a la realización de sinergias interinstitucionales y al trabajo en redes; ciertos impulsos que llevan a pasar del nacionalismo al universalismo, de los prejuicios étnicos y culturales a la tolerancia y, del pluralismo y los totalitarismos, a la democracia; la creciente conciencia convertida en nuevo valor respecto de la biodiversidad y, por fin, una sensibilidad ecológica que define el mundo como casa propia que debe ser cuidada por sus moradores.

A pesar de la insistencia de ciertos autores en la inutilidad de iniciar cualquier reflexión educativa haciendo referencia a los últimos cambios operados, al ser ello considerado un tópico (Carbonell 2008: 7), por nuestra parte si se contempla tal posibilidad desde la necesidad de contextualizar históricamente la labor docente. En tal sentido creemos fundamental tener en cuenta la influencia que, tanto la posmodernidad como la globalización, continúan ejerciendo sobre la educación y la formación del profesorado. Convicción esta que sostenemos desde la necesidad de ser consecuentes con tal realidad sin limitarnos a actuar como sujetos pasivos de esa doble tendencia y aceptando el reto de adoptar actitudes ciudadanas y profesionales que contribuyan a amortiguar los efectos negativos que ello ha conllevado. Es más, centrados de modo particular en la realidad de la globalización, reconocemos sin lugar a dudas que 
los cambios operados recientemente han repercutido en políticas y en prácticas e instituciones educativas obligando a readaptar no pocos de los objetivos asignados a las mismas.

No cabe duda de que en tales circunstancias las finalidades educativas deben orientarse no sólo a la necesidad de enseñar, sino también a la urgencia de aprender a coexistir en espacios diversos, de elaborar un sentido de la identidad viable en contextos multiculturales y de adaptarse a una nueva cultura regida por la información y la comunicación como elementos a considerar. Y, siempre, rescatando el gran tema olvidado durante las últimas décadas: la cuestión del sentido. Desde nuestro punto de vista la esperanza en la globalización cultural reside en la necesaria extensión de los valores democráticos y los derechos humanos, junto con todas las finalidades educativas enumeradas; empeño en el cual la escuela pueden jugar un papel esencial desde sus posibilidades para contribuir al desarrollo de una comunidad ética en búsqueda de la igualdad social y del sentido.

Siendo optimistas pensamos con otros autores (Fernández, 2001: 14) que, en sociedades cambiantes la escuela puede y debe actuar como instrumento de transformación al encontrase inmersa en desconcertantes situaciones que ha derivado en una manifiesta crisis de la insostenibilidad de dinámicas anteriores que ha dado paso a todo un escenario lleno de nuevas oportunidades.

\subsection{Formación inicial del profesorado en un mundo globalizado}

Somos conscientes de que hoy por hoy toda acción relacionada con la formación de los futuros docentes debe asumir que las implicaciones derivadas del hecho de encontrarnos inmersos en un creciente proceso de globalización conducen hacia una inevitable reflexión sobre ello. Postulado a partir del cual la primera constatación que realizamos es que son muchas las definiciones posibles de globalización; tantas, como diversas son las ópticas aplicables a la compresión de un complejo fenómeno que ofrece múltiples dimensiones que interactúan entre sí y parten de la liberalización económica y la mundialización financiera y del capital, apoyada en la continua innovación tecnológica y el predominio de la cultura occidental.

Juzgamos que una realidad con tales connotaciones debe ser analizada como un proceso histórico de larga gestación que caracteriza el desarrollo de la humanidad desde la Antigüedad, pero que nunca se reveló con la actual magnitud (Bifani, 2002). Ello hace que el término se asocie a un fenómeno dinámico y a una idea de cambio que, por su vertiginoso recorrido, exige una reflexión crítica sobre las formas en que se manifiesta y las consecuencias negativas de ellas derivadas en relación a los procesos de exclusión y marginación de aquellos sectores de la población y países a quienes resulta imposible sumarse a la dimensión tecnológica del fenómeno. Asociados a tal realidad, han surgido conceptos tales como:

10. La sociedad de la información a la que M. Castells (1997 y 2001) se refiere como modelo de desarrollo basado en la expansión y circulación de información y entretenimiento digitales.

$2^{\circ}$. La sociedad del conocimiento. Así reconocida por P. Drucker (1993 y 1994) al considerar el conocimiento como el recurso económico básico de la nueva sociedad.

$3^{\circ}$. La sociedad postindustrial. Categoría acuñada en los años setenta del S. XX por el sociólogo americano D. Bell para describir el cambio económico de la economía industrial, basada en la producción de bienes y la concentración de mano de obra en el sector servicios y comunicación. 
$4^{\circ}$. La postmodernidad. Concepto aludido que, en términos culturales, refleja el avance imparable de una economía basada en el conocimiento y en la convicción de que la investigación y el desarrollo científico-tecnológico son estratégicos.

Todo esto indica hasta qué punto el fenómeno de la globalización incluye procesos que trascienden lo económico, financiero y tecnológico, para manifestarse en ámbitos políticos, ideológicos, sociales y culturales que se convierten en obligada referencia para toda propuesta educativa medianamente actualizada. La creciente concentración de poder económico mundial, con fusiones y adquisiciones, necesita de instituciones supranacionales para gestionar sus efectos, así como de poderes transnacionales que tomen decisiones al respecto. Esto conlleva que las funciones tradicionales de los estados y su forma de entender la soberanía se modifiquen al demandarse nuevas formas de gobernabilidad.

Mientras la globalización se justifica desde ideologías diversas que la instrumentalizan al servicio de sus intereses, el fenómeno provoca también la difusión de tales ideas por todas partes y que estas adquieran una nueva dimensión en que los valores se globalizan igualmente. Para muchos resulta primordial que la mundialización de la economía y el vertiginoso cambio tecnológico vaya parejo al respeto universal a los derechos humanos o la globalización de tales derechos (AA.VV., 2003). No obstante todo ello ha sido acompañado por fuertes críticas respecto a su presentación estándar ante las naciones y culturas que exigen revisar su formulación frente a nuevas situaciones como los movimientos migratorios, el creciente aumento de refugiados o los actuales movimientos anti sistema; manifestaciones todas ellas que replantean nuestras definiciones de identidad y cultura.

Para algunos, el concepto representa la visión universalista de la ciudadanía, entendida como vínculo moral y racional que une por encima de fronteras (Acosta, 2001); acción en que, tanto los medios de comunicación clásicos como las nuevas vías de información, han desempeñado una gran labor al abrir posibilidades desconocidas para el ejercicio de la ciudadanía en el marco de un nuevo contexto cosmopolita y multicultural (Mussbaum, 1999). No obstante, la esclavitud de la estética y el consumo lanzan a veces una frívola visión de la misma.

La imagen y el lenguaje audiovisual, en su dimensión mercantil, descontextualizan y reinterpretan una realidad exenta de conflicto, de ahí que para algunos sea necesario rescatar el concepto de educación global como convivencia entre distintas culturas e idiomas y no sólo como violencia cultural (Mulet, 2003). Ello demuestra, también, que la profusión de información no ha contribuido a que ésta sea de mayor calidad y veracidad ni a que sirva para ordenar mejor las ideas, sino que ha primado el entretenimiento y un pensamiento mínimo que requiere ser contrarrestado mediante una guía moral o intelectual.

Esbozado someramente el fenómeno de la globalización como entorno inmediato de toda reflexión relativa a la actual formación del profesorado, es necesario dar un paso más señalando algunos de los problemas planteados por ella junto con el papel del conocimiento en su resolución. Lo primero que ha de subrayarse al respecto es que la sociedad del conocimiento se caracteriza por el peso que en ella obtiene la innovación derivada de la investigación para el desarrollo, de ahí que el recurso básico de la misma no sea el capital o la mano de obra sino el conocimiento. En ella, la producción se relaciona con el consumo; hecho que conlleva la incesante construcción de culturas y sistemas de innovación con una velocidad e inteligencia y una capacidad de anticipación al mercado sólo propia de espiritus emprendedores. Por ello, en el nuevo modelo de sociedad del que formamos parte unido a la generación, procesamiento, dominio y propagación 
del conocimiento se convierte en fuente de riqueza y transformación de las actividades productivas (Castells, 1997: 47).

Según A. Hargreaves (2003: 28) la sociedad del conocimiento presenta tres dimensiones: Una circulación y procesamiento del conocimiento y la información complejos; unos equipos profesionales dispuestos a promover la innovación continua de productos y servicios; y una esfera educativa, técnica y cientifica. El papel central que juega el conocimiento en la nueva economía globalizada es reconocido también por M. Carnoy (1999) cuando afirma que en esta sociedad resulta clave no sólo el acceso a la información sino, sobre todo, su procesamiento; razón por la cual podría hablarse de una sociedad del aprendizaje basada en una cultura de la innovación continua y la creatividad, capaz de afrontar los problemas de un mundo interdependiente y complejo. Por ello la escolarización y la enseñanza exigen trabajar centrados en el aprendizaje y la creación de conocimiento.

Estos incesantes cambios sociales exigen que la educación sea una experiencia global que dure toda la vida, en función del enriquecimiento continuo de los conocimientos. Educar, más que nunca, sirve para vivir en una futura sociedad del conocimiento, que requerirá de una gran flexibilidad, capacidad de adaptación y control de la incertidumbre valorando al mismo tiempo las habilidades sociales, la capacidad para aprender a aprender junto con la facultad de seleccionar la información creciente e incontrolada de los medios de comunicación.

A su vez, la globalización nos enfrenta a desafíos que requieren una solución interdisciplinar y multidimensional. La articulación mundial del mercado, gracias al desarrollo científico y tecnológico, ha contribuido a que el progreso no sea extensible a todo el planeta al contribuir, cada vez más, al aumento de las desigualdades, el desarraigo de los inmigrantes y la amenaza al medio ambiente a través de fenómenos de urbanización desordenada; tendencias que desembocan en una crisis social que ha alterado las antiguas solidaridades desencadenando fenómenos de emigración, exclusión, marginación y contestación social, violencia y delincuencia como hondas fracturas que aun deben afrontarse.

Según A. Touraine (2005) esta situación ha relegado las relaciones sociales a favor de la economía, haciendo decaer el interés por lo social y la pérdida de sentido de las instituciones que ignoran cómo responder a ello, traduciéndose todo en un debilitamiento de la democracia. Bajo tal circunstancia, las redes de representación social carecen de efectividad siendo cada vez más importante el volumen de población de origen inmigrante sin integrar generando tal circunstancia una tendencia a la individualidad y a la vinculación a nuevas identidades definidas por el género, la raza, la religión o la lengua. Si para dicho sociólogo francés esta situación hace vislumbrar un nuevo escenario de enfrentamientos sociales, la esperanza sólo reside ya en el sujeto que busca sentido a su existencia frente a la realidad de un mundo postsocial.

Sin embargo no todos los pronunciamientos se producen en el mismo sentido, pues la mayoría de las voces apuestan por una solución más amplia que la individualista de Touraine. Otros aventuran una neutralización de los efectos de la globalización que pasa por la introducción de relaciones más equitativas bajo principios éticos basados en el respeto a los valores de otras culturas, posición en que el conocimiento del otro, como ser diferente, tendrá un componente principal. Por otro lado resulta obligado reconocer que existen opiniones contrarias a considerar el problema desde el componente cultural; posturas desde las cuales las dificultades de integración y cohesión social se vinculan más a factores económicos y 
sociopolíticos (Carbonell, 2005: 29). En todo esto consideramos que la educación jugará un papel fundamental, construyendo un espacio de socialización caracterizado por el pluralismo cultural e iniciando a los futuros ciudadanos en valores integradores frente al avance de prejuicios raciales y manifestaciones de violencia social. De nuevo, como en todas las etapas de nuestra historia reciente, la educación en valores se ha desvelado como el antídoto fundamental contra la intolerancia; en éste caso, mediante un acercamiento a la historia y a la cultura de aquellos que son diferentes.

El primer obstáculo en tal sentido surge al constatar que los sistemas educativos continúan reproduciendo moldes culturales monolíticos más interesados en el desarrollo del conocimiento abstracto en detrimento del desarrollo integral de la persona. No obstante, cada vez son mayores las valoraciones sobre el papel fundamental que debe jugar la escuela en la integración de la población inmigrante y desarraigada, dado que puede ser factor de cohesión de gran importancia mientras el fracaso escolar de la misma no contribuya a generar y aumentar la exclusión social. Pero hemos de reconocer que las desigualdades entre clases sociales y grupos étnicos, sumadas a la invisibilidad del género no mejoran la situación (Rambla y Bonal, 2006). De igual forma, la exclusión social y discriminación escolar presenta otras facetas, muy variadas que afectan a amplias categorías sociales, ya sea por la ruptura de los vínculos sociales entre el individuo y la sociedad, por las diferencias sociales, la división económica del trabajo y la separación de esferas o por un orden social coercitivo impuesto a través de relaciones jerárquicas de poder (Luengo, 2005).

Si esta es la situación en Occidente, una visión del resto del mundo no resulta más tranquilizadora pues la desigualdad educativa es otra de las manifestaciones de la globalización. Al respecto, los organismos internacionales alertan sobre la situación en África y algunas zonas de lberoamérica como ejemplos sangrantes de las diferencias territoriales cada vez más profundas en el planeta. La realidad de estos países indica que la posibilidad de aproximarse al mundo desarrollado es cada vez más remota, situación acentuada por la progresiva privatización de la educación en nuestro entorno y en países menos desarrollados (Feldfebler y Verger, 2006), puesto que ello implica profundizar en las desigualdades, cuando precisamente las políticas educativas apuntan hacia la necesidad de generalizar y ampliar la permanencia en el sistema educativo (Rambla, Valiente y Constans, 2006).

En esa tarea, la escuela -reflejo de la variedad multicultural de la sociedad- ha de fomentar el ejercicio de la vida social. La enseñanza, para ser coherente con tal necesidad debe asumir el pluralismo social existente y la riqueza cultural que ello le brinda. En tal sentido, la propuesta de A. Touraine y su esperanza en el individuo adquiere sentido, puesto que la escuela ha de respetar la singularidad de cada niño acogiéndolo para socializarlo mientras desarrolla su propia personalidad en función a su cultura de origen y, todo ello, haciéndole crecer en sus relaciones interpersonales y en situaciones sociales que lo vinculen con el grupo (Casals, 2006). Por tanto, esa escuela debe fundarse en el diálogo y en la comunicación para contribuir a una renovada visión cívico-social. Ahora más que nunca, educar desde las CCSS debe ser el instrumento privilegiado de ayuda a las personas en su preparación para una vida plena, una ciudadanía participativa, una posición económica digna, una convivencia no conflictiva, una apreciación adecuada de la cultura y unas relaciones sociales en cambio permanente.

No cabe duda de que la educación es una herramienta que favorece la igualdad de oportunidades (Bonal et al., 2006) y que ello exige compensar las desigualdades por razón de clase, cultura o género al tiempo que ofrecer respuestas a los retos que le plantea la sociedad del conocimiento. Si hasta hoy se ha 
hablado de las actitudes y valores que han de promoverse en la institución escolar en relación a la necesidad de redefinir una nueva ciudadanía democrática, esos valores y actitudes también deben contribuir a afrontar los desafíos de una nueva economía. Aquí deben invertirse los términos considerando en primer lugar la relevancia de los problemas sociales y en segundo los económicos. A pesar de todo las reformas educativas continúan prestando mayor atención a los contenidos matemáticos y a la lectoescritura, desde una inquietud por el alto grado de procesamiento de información y conocimiento de nuestra sociedad, olvidando el desarrollo personal y social del niño con vistas a su integración; situación indicativa de hasta qué punto aún se continua primando el aprendizaje cognitivo y relegando el emocional, que sería de gran interés para el desarrollo de una identidad cosmopolita como señala A. Hargreaves (2003).

No obstante, resulta imposible negar los nuevos retos planteados a la sociedad de la información o del conocimiento. La educación adquiere en ella mayor importancia al representar la cualificación profesional un papel decisivo en la economía, pero también resulta vital desarrollar en el alumnado competencias intelectuales para afrontar el continuo progreso técnico en que vivimos inmersos. Por ello consideramos que debe abogarse por competencias específicas que aúnen conocimientos teóricos y prácticos y faciliten la resolución de conflictos así como por la comunicación ante las nuevas formas de socialización desencadenadas y una definición de nuevas identidades individuales y colectivas que contribuya a favorecer la comunicación.

En la nueva forma de organización social y económica, el conocimiento surge como la variable fundamental en que la información y capacidad para producir y manejar conocimiento se manifiesta como recurso esencial. En tal contexto, según recordó Tedesco (1995: 22), la educación como actividad productora y distribuidora de conocimiento, asume una gran importancia desde el punto de vista político-social (desde el presupuesto de que quienes están involucrados en la producción y la distribución de conocimiento desempeñarán un papel fundamental en la generación de conflictos y su solución) y desde la óptica de los contenidos (desde la convicción de que debe trabajarse por evitar la separación entre conocimiento y pensamiento por constituir ello una dificultad para entender, pensar y hablar de aquello que, sin embargo, es posible).

El pensamiento separado de la actividad, la investigación alejada de la acción y el sujeto apartado del objeto no son buenos referentes. El sujeto debe caminar hacia el conocimiento en su búsqueda de identidad y el reconocimiento de los otros. La globalización de esta era planetaria precisa replantearse la concepción de los problemas en esa misma escala contextual. La violencia, la explotación, la exclusión o la falta de sentido exigen para su comprensión un pensamiento complejo pues, como recordaba E. Morin: "El problema de la comprensión es crucial, debe ser una de las finalidades de la educación para el futuro" (2004: 113).

La educación debe profundizar en esos problemas y para ello ha de adentrarse en el conocimiento de la condición humana, con su identidad compleja. Ha de comprender las incertidumbres, los fanatismos desde una visión multidimensional que aúne el conocimiento de las partes y el de la totalidad, sin eludir responsabilidades ni la adopción de posturas solidarias. Por ello, según este autor, la educación futura requiere el desarrollo de un pensamiento complejo mediante una concentración de conocimientos que ubique la condición humana en el mundo. Asimismo piensa que debe favorecerse la aptitud natural para plantear y resolver problemas empleando la inteligencia, movilizando la cultura cientifica y las humanidades, 
circunstancias que nos harán aptos para responder a los desafios de la globalidad y de su complejidad (lbídem, 41 y 58).

Para esto Morín considera necesaria una reforma del pensamiento que tenga como fin la comprensión de lo complejo y ello sólo es posible desde un conocimiento contextualizado y apto para responder a los problemas, enfrentar las incertidumbres y comprender al género humano (Ibídem, 122). Tal reforma exige el cambio de la educación desde la universidad planteando como alternativa ante la pérdida de sentido de la misión social del maestro una renovación de las ideas, la integración de las disciplinas en torno a grandes temas y su vinculación a la sociedad. Un currículo integrado que facilite una comprensión reflexiva y crítica de una realidad social cambiante (Torres, 1998). La meta será que conocimientos parciales aborden problemas globales promoviendo una inteligencia general y la comprensión de lo complejo (Miralles, 2005). Vía por la cual los objetivos de la formación del profesorado han de pasar por procurar corregir desde nuestra área algunos de los vacios existentes mediante la implementación de métodos y estrategias adecuados para apoyar el desarrollo de un conocimiento eficaz, abordar la complejidad de lo real y la hondura de la condición humana mediante estrategias que ayuden a afrontar de formar adecuada las incertidumbres, la asunción de la realidad en todas sus dimensiones y el descubrimiento de una ética universal.

\section{Retos formativos y responsabilidades docentes}

Los problemas actuales de la Educación se encuentran determinados por el contexto social en que nos hallamos inmersos, circunstancia que convierte la labor docente en un asunto de gran complejidad, sobre todo si se busca dar respuesta a la diversidad. Las necesidades educativas planteadas por esta sociedad del conocimiento exigen que una función tan desvalorizada como la docente se transforme en una profesión del aprendizaje no desvinculada de la vocación social que siempre ha manifestado.

Según A. Hargreaves (2003: 20) los docentes se encuentran en una situación de intereses contrapuestos que les convierte en catalizadores de esta sociedad del conocimiento y de las oportunidades que ella promete. Por esto se espera de ellos que sean el contrapunto de sus amenazas, cuando en realidad no son más que víctimas de las crecientes expectativas creadas por una educación que solo ofrece soluciones estandarizadas. Frente a tal empobrecimiento educativo se propone trabajar por hacer comunidad profesional, apostar por el trabajo en equipo y la formación permanente con el fin de afrontar los problemas propios de situaciones educativas cambiantes y emprender la mejora continua basada en la investigación. Retos que convierten sin duda esta tarea en una actividad plagada de obstáculos derivados de la inercia que embarga gran parte de la cultura académica universitaria.

Si se parte de recientes análisis sobre la forma de desarrollar la profesión docente se hallarán escasas razones para el optimismo. Para algunos el futuro es poco halagüeño pues sus estudios continúan nutriéndose de aspirantes procedentes de clases sociales populares al tiempo que la profesión continúa gozando escaso prestigio social. Fenómeno al que se añade el hecho de que la Universidad, al menos en bastantes lugares de Europa, forma a un elevado número de titulados que no podrá desempeñar esa labor; situación que aviva la eterna demanda de potenciar el componente profesional de la formación y trabajar sobre la competencia profesional. Ello implica un conjunto integrado de conocimientos, habilidades, actitudes y valores que componen la compleja cultura profesional del docente para cuya formación se 
requiere un delicado y creativo equilibrio de acción y reflexión, práctica y teórica, experiencia tutelada y reflexión alejada de la formación academicista.

Esto puede ser así considerado porque la tarea profesional del docente consiste a nuestro juicio en provocar, orientar y acompañar en el aprendizaje e investigar y diagnosticar permanentemente para elaborar estrategias de intervención que mejoren su práctica educativa. Tal meta sólo puede alcanzarse mediante una formación cultural amplia, basada en la práctica, realizada desde ella y para ella, apta para impulsar las capacidades básicas de aprender a aprender y a pensar, acompañada de principios eficaces para vertebrar el currículo de formación en una sociedad de la información y del conocimiento. En tal sentido son muchos los que abogan por un currículo basado en la resolución de problemas pedagógicos para desarrollar el pensamiento práctico durante la formación inicial (Pérez Gómez et al., 2004); propuesta que por nuestra parte concretaríamos mediante la implementación desde la Didáctica de las CCSS de en unos contenidos curriculares orientados a:

$1^{\circ}$. Analizar problemas pedagógicos desde los conceptos y teorías de las disciplinas educativas.

$2^{\circ}$. Abordar diseños de intervención desde la cooperación en el proceso de aprendizaje, estimulando el contraste de opiniones, perspectivas y enfoques.

$3^{\circ}$. Fomentar una práctica democrática desde el respeto a la pluralidad de perspectivas, planteamientos, enfoques y proyectos.

$4^{\circ}$. Formular las actividades de enseñanza y aprendizaje como estrategias de investigación y experimentación curricular en contextos escolares complejos.

$5^{\circ}$. Estimular la autonomía profesional del docente como expresión del carácter artístico, indeterminado e inacabado de todo proyecto educativo.

$6^{\circ}$. Analizar el contexto social como factor clave en la orientación, sentido y potencialidad que adquieren los fenómenos educativos en cada grupo humano.

La formación del profesorado se revela de nuevo en tales circunstancias como clave fundamental de una educación de calidad. Por ello reconocemos con J. Gimeno (2005: 61) que la comprensión de un mundo gradualmente complejo requiere el ejercicio del papel de mediador entre lo conocido, el docente y otros conocimientos elaborados procedentes de diversas fuentes junto con la oferta de diversas valoraciones sobre los mismos. A pesar de ello, desde la actual realidad ha de reconocerse que los cambios sociales experimentados ofrecen un carácter intrageneracional al tiempo que radican en aspectos fundamentales de la experiencia humana; razón por la cual demandan una formación permanente capaz de asegurar el aprendizaje de los conocimientos finalmente útiles y aplicables en el trabajo y la vida social. Constatación junto a la ha de subrayarse cómo a la formación inicial, que pierde gradualmente peso, le continúa correspondiendo el desarrollo de las capacidades generales para aprovechar posteriormente sus posibilidades (Fernández Enguita, 2001: 21).

La formación superior es hoy reconocida, por su impacto en el sistema productivo, como motor de desarrollo económico, pero también por su poder para contribuir a los problemas que desencadena la nueva sociedad global. Por ello resulta imposible no ver la Universidad como depositaria y creadora de conocimientos, instrumento de transmisión de experiencia cultural y científica, reconociendo al mismo tiempo su papel crítico ante problemas de carácter ético y cientifico. De hecho, en el Informe Delors (1996: 
166) las funciones esenciales asignadas a ella son: Preparar para la investigación y la enseñanza, ofrecer formación especializada y adaptada a las necesidades sociales, abrirse a todos para responder a la educación permanente y fomentar la cooperación internacional.

Las recientes reformas en los programas de formación de países del entorno europeo han reconocido la necesidad de aumentar los años de formación inicial y del grado académico, al tiempo que tienden hacia una especialización y profesionalización de los estudios universitarios de Magisterio, apostando por modelos simultáneos frente a los sucesivos reconociendo, no obstante, que en ambos la formación debe ofrecer un componente eminentemente práctico ${ }^{2}$. En el caso de España la formación inicial y permanente del profesorado ha recibido una atención prioritaria por el imperativo de su adaptación al contexto del nuevo Espacio Europeo de Educación Superior, y la necesidad de aumentar la valoración social de la función docente. En tal sentido se reconoce que la formación inicial debe incluir preparación científica, pedagógica y didáctica que se completará con la tutoría y asesoramiento al profesorado en formación por parte de compañeros experimentados. Según esto la formación no sólo debe centrarse en las destrezas a desarrollar, sino también en la dimensión moral y ética de la futura labor docente, no renunciando a lograr una educación mundializada que frene los efectos de la globalización. Por ello la Universidad ha de responder, además de al sistema productivo, a las nuevas demandas planteadas a la educación en general y a la formación del profesorado en particular.

Probablemente, el contexto académico universitario no sea el lugar más adecuado para desarrollar habilidades y destrezas que posibiliten este proceso, que sólo puede alcanzarse con la práctica, no obstante, siempre será conveniente su iniciación en ellas. En tal sentido, las teorías psicológicas y pedagógicas, así como las didácticas específicas, han de enfocarse hacia la reflexión sobre la implicación moral de la educación y la mejora de los métodos de enseñanza pues siempre es necesario comprender e interpretar el sentido de las decisiones curriculares frente al posible reduccionismo o tecnicismo al que puede conducir el desarrollo de competencias. El camino puede ser la profundización en la reflexión crítica sobre la eficacia de la situación de transmisión, preguntándose por su enseñanza desde el punto de vista del aprendizaje efectivo del alumnado, en función a su diversidad.

La misión del maestro ha dejado de ser la acción de un agente cultural, transmisor de moral y valores, para transformarse en otra actividad más profesional (Carr 2005: 102). Por ello, la labor docente debe abrirse aún más a la realidad actual; exigencia que lleva implícita la preocupación por fenómenos como la globalización y los problemas por ella ocasionados en el ámbito de la cohesión social. La educación, entendida como ayuda al alumno para crecer en sabiduría y discernimiento moral, debe contribuir a la integración social y ello exige la formación de un profesorado sensible a ello. Asimismo no son pocos los autores que piden vincular a los maestros en formación a los problemas de la sociedad con el fin de que ofrezcan su traslación a la enseñanza desde una concepción del conocimiento no sólo limitada al desarrollo de competencias sino también a los valores, con objeto de ejercer su función del modo más completo posible. A partir de tales premisas la mera transmisión de información y conocimientos no basta, pues debe apostarse por el diálogo y la presentación realista de un contenido formativo bien situado en su

2 J. M. Esteve (2003). La diferencia estriba en que los modelos simultáneos diseñan títulos profesionales específicamente dedicados a la docencia, integrando formación científica y psicopedagógica, mientras los sucesivos exigen una formación científica previa a la profesional. 
perspectiva histórica para que el futuro docente pueda contribuir a mejorar su visión de la educación y desarrollar su sentido crítico además de sus actitudes profesionales y ciudadanas.

Desde el ámbito norteamericano, P. G. Avery (2004) aboga en la formación del profesorado por trabajar la identidad cívica en una sociedad interdependiente, multicultural, pluralista y global. Juzga fundamental tal asunto porque a través de la enseñanza de los Social Studies los jóvenes descubren un camino para adquirir conocimiento y habilidades cívicas. Ello obliga a considerar tres cuestiones indisociables: una perspectiva global, problemas medioambientales y de derechos humanos y la tolerancia respecto a la diversidad de creencias. En función de ello, J. A. Whitson (2004), valora que la formación del profesorado de Social Studies debe responder a la necesidad específica de promover su competencia cívica.

La cuestión no ofrece una definición simple si se tiene en cuenta que la aportación de las CCSS y la formación cívico ciudadana se encuentra en transición desde una dimensión local hacia otra global que define al buen ciudadano como el poseedor de una visión de futuro interesado en la preocupación inmediata de ejercer el voto, por problemas medioambientales o por el respeto de los derechos humanos a nivel mundial (Avery, 2004: 38). Ello conduce a una concepción de la ciudadanía que reconoce al individuo en relación a los otros y como parte de una sociedad interconectada y global (lbídem, 41). Perspectiva desde la que Kubow, Grossman, Ninomiya (2000) insisten en que el ciudadano del S. XXI debe poseer el siguiente elenco de cualidades en cuyo desarrollo puede resultar muy significativa la contribución de la Didáctica de las CCSS (Ibídem, 40): Habilidad para comprender, aceptar, apreciar y tolerar diferencias culturales siendo sensible a la defensa de los Derechos Humanos; capacidad para pensar de forma crítica y sistemática y buena voluntad y pericia para participar en política local, nacional e internacional; habilidad para trabajar de forma cooperativa y asumir la responsabilidad de sus deberes sociales y disposición para resolver conflictos de forma no violenta; y capacidad para aproximarse a problemas mundiales como miembros de una sociedad global y predisposición para cambiar su estilo de vida y hábitos de consumo para proteger el entorno.

A su vez las cualidades de las que debería ser portador un ciudadano democrático en un contexto global -también susceptibles de implementación desde esta misma área de conocimiento- se manifestarían en niveles tales como (Ibídem, 2004: 42): El conocimiento de los principios de la democracia, la estructura y función del gobierno, líderes y organizaciones políticas; el dominio de las técnicas (tecnologías, pensamiento crítico y sistemático, resolución de conflictos) y las conductas (obligaciones políticas como votar o participar en actividades comprometidas); y la estima de los valores (tolerancia y cooperación, derechos humanos y problemas medioambientales. etc.). Todo esto resulta útil para entender que una formación docente completa y un ejercicio pleno en un contexto global requieren ciertas habilidades de comunicación junto a determinadas capacidades para desarrollar un pensamiento crítico, resolver pacíficamente los conflictos y adquirir actitudes tolerantes además de una preocupación por los derechos humanos y los problemas medioambientales debiendo asumir en todo ello una clara responsabilidad la Didáctica de las CCSS; área desde la que podrá trabajarse por diversas vías la perspectiva global en la formación del profesorado mediante la discusión sobre problemas sociales y políicos. 


\section{Aportaciones de la Didáctica de las CCSS a la formación del profesorado.}

Teniendo en cuenta lo anterior, consideramos que el Área de Didáctica de las Ciencias Sociales puede y debe contribuir al proceso formativo inicial de los futuros maestros de Educación Primaria, básicamente, mediante dos aportaciones: Su propia identidad y definición como disciplina y las finalidades que, como ámbito de conocimiento específico, le son propias y persigue desde el contexto más amplio del saber humanístico y social.

Desde su identidad y definición cómo campo especifico de conocimiento y materia de enseñanza, la primera contribución que la Didáctica de las Ciencias Sociales consideramos que realiza al proceso de la formación inicial del profesorado de Ed. Primaria y Secundaria consiste en su propia realidad como disciplina científica; dimensión esencial de la misma que nos lleva definirla como: $1^{\circ}$ Área de conocimiento universitario encuadrada en el campo de las Didácticas Específicas; $2^{\circ}$ Saber cientifico así definido por su objeto y metodología; $3^{\circ}$ Ámbito de conocimiento desplegado históricamente en diferentes paradigmas epistemológicos que, con el tiempo, se ha concretado en diversos modelos de formación del profesorado; $4^{\circ}$ Campo del saber que puede y debe contribuir a la formación de profesores reflexivos; $5^{\circ}$ Saber integrador que, a título ilustrativo, podría concretarse en tres dimensiones específicas:

a) Su capacidad como fuerza "catalizadora" en la confluencia de disciplinas del ámbito de las Ciencias de la Educación (Psicología, Pedagogía, Teoría e Historia de la Educación, Sociología, Antropología y Didáctica general) y sus materias referentes (Historia, Geografía y Arte) estableciendo relaciones reciprocas e interdisciplinares entre sus paradigmas y variadas aportaciones.

b) La dimensión integradora propia de los procesos unitarios de Enseñanza-Educación desarrollados por las CCSS; realidad que confiere un carácter unificado y unificante a todos los procesos que le son propios Icognitivos-categoriales, valorativos-morales y sociales e históricos $)^{3}$.

c) El valor educativo de que son portadoras las CCSS en los procesos de Enseñanza-Aprendizaje en orden, entre otros objetivos, al logro de aprendizajes significativos, la mejora del conocimiento del Medio y el desarrollo de actitudes humanas y cientificas -activas y críticas- de respeto hacia el mismo y hacia las personas, asi como al inicio en el uso de técnicas y procedimientos propios de las $\operatorname{cCSS}^{4}$.

Desde sus finalidades propias como área de conocimiento juzgamos que el segundo elemento que puede ayudar a la compresión de la aportación de la Didáctica de las CCSS a la formación del profesorado vendría a ser la enumeración y análisis de las grandes finalidades de la Educación Social. En tal sentido cabría reflexionar sobre el enorme potencial de las Ciencias Sociales más presentes en el curriculum de tales niveles educativos -en particular la Historia, la Geografía y el Arte y además, al menos en España y en el

\footnotetext{
${ }^{3}$ C. Aranguren (1999) lanzó esta hipótesis inicial hace más de una década que la misma autora ha confirmado con posterioridad constatando cómo toda aproximación del alumnado a la realidad objeto de estudio siempre plantea a éste múltiples interrogantes y problemas tanto desde la esfera de las elaboraciones conceptuales, como desde las condiciones valorativas que representa el objeto de conocimiento.

4 Fue esta una cuestión reivindicada hace años por A. Liceras (1993: 30-33) que consideramos de total actualidad. Es más, a pesar de que tal autor la aplicase al estudio del Conocimiento del Medio, juzgamos que su aportación ofrece total aplicación a los elementos propios de las diversas CCSS incluidos en el curriculum formativo del Grado de Maestro en Ed. Primaria.
} 
resto del EEES a excepción de Francia, el estudio del Hecho Religioso-, canalizadas mediante su proyección didáctica, para:

- Suscitar actitudes de comportamiento cívico-democrático posibilitando así las ineludibles condiciones de una formación integral capaz de garantizar el pleno desarrollo de la personalidad humana. Y todo ello desde la conciencia de las dificultades existentes para definir las finalidades educativas en un espacio saturado de conflictos derivados de la permanente confluencia de intereses ideológicos diversos.

- Superar la mera adquisición de conocimientos factuales y memorísticos evitando a la vez el riesgo de caer en procesos de adoctrinamiento ideológico y trabajar sobre programas no cerrados.

- Contribuir a un proceso educativo en valores que posibilite al alumnado construir un sistema de significados y desarrollar actitudes en sintonía con los valores de racionalidad, libertad, igualdad, participación, respeto a la pluralidad de identidades, conservación del legado natural y cultural recibido cimentándose todo sobre el necesario equilibrio entre la dimensión inmanente y transcendente del ser humano.

- Asumir el reto de la actual situación de cambio de valores, formar en un pensamiento crítico desde la consciencia de la escasa predisposición de los alumnos para pensar- y educar para la solidaridad y el respeto a la diversidad.

\section{Didáctica de las CCSS y formación de profesores en la Universidad de Granada}

Desde una voluntad descriptiva respecto al lugar ocupado por la Didáctica de las CCSS en el Grado en Magisterio de Primaria recientemente implantado en la Universidad de Granada, la primera constatación que puede realizarse en el conjunto de la universidad española es el hecho de que dicha materia ha comenzado a impartirse en un contexto organizativo cuyo potencial docente se distribuye de forma bastante variable en cada centro universitario. Ofrecida bajo denominaciones diversas, su presencia oscila entre los 15 créditos de la Universidad de Granada y los 18 de la Complutense de Madrid, enmarcada siempre entre el "Bloque de Formación Didáctico Disciplinar" y el de materias optativas, y distribuida su carga docente en créditos de varias disciplinas propias del área, según el criterio de cada institución universitaria.

En el caso de la Universidad de Granada la materia se encuadra en el "Bloque de Formación Didáctico Disciplinar" integrando el Módulo "Enseñanza y aprendizaje de las CCSS" cuyos 15 créditos se distribuyen en dos materias diferentes: El Patrimonio Histórico y cultural y su Didáctica con 6 créditos y Didáctica de las CCSS con 9. En universidades como las de Cádiz o Jaén, estas materias se denominan Didáctica de las CCSS I y Didáctica de las CCSS II. La de Málaga imparte un total de 20 créditos distribuidos en Educación para la ciudadanía y los Derechos humanos, con 6 créditos, Didáctica de las CCSS, con 8, y Didáctica de la Cultura Andaluza con 6. En la Universidad de Salamanca se denomina: Enseñanza y aprendizaje de CCS I, II y III, contando con un total de 18 créditos. Otros centros como la Universidad Complutense de Madrid aumentan ampliamente su oferta al brindar en su Grado de Maestro en Ed. Primaria las siguientes materias básicas y obligatorias: Fundamentos y Didáctica de Geografía, 6 créditos, Fundamentos y Didáctica de la $H^{a}, 6$ créditos y Fundamentos y Didáctica de la $H^{a}$ del Arte, 6 créditos; 
materias a las que se suman otras tres más como optativas: Geografía de España y su Didáctica, 6 créditos, $H^{a}$ de España y su Didáctica, 6 créditos, $H^{a}$ del Arte Español y su Didáctica, 6 créditos.

Aunque no es la finalidad de este trabajo abordar un análisis comparativo del tratamiento obtenido por la Didáctica de las CCSS en las 74 universidades existentes en España -22 privadas y 52 públicas-, en una aproximación inicial al tema, si podrían realizarse algunas constataciones. Así, en primer lugar se verifica que la presencia de la Didáctica de las Ciencias Sociales en el cuadro de materias del Grado de Maestro de Ed. Primaria oscila entre un $6.25 \%$ y un $7.5 \%$ del total para el bloque de materias de formación básica y obligatoria y un $0 \%$ y un $7.5 \%$ para el de materias optativas, siendo exponente cada uno de tales porcentajes de la sensibilidad y posibilidades existentes en el seno de los respectivos centros universitarios. Valgan pues estos datos como un primer acercamiento al tema que enmarque nuestro objeto de estudio.

Dando un paso más en la descripción del actual perfil de la materia Didáctica de las Ciencias Sociales, según quedó configurada en el año 2010 entre el conjunto de asignaturas y materias del Grado en Educación Primaria de la Universidad de Granada, merecería la pena recordar algunos de los principales elementos que la caracterizan. Para ello extractaremos parte de la información que sobre la misma se incluye en el apartado de módulos y materias de la Memoria de este Grado y en la Guía Docente de la misma elaborada por el Departamento de Didáctica de las CCSS de la Facultad de Ciencias de la Educación de esta universidad ${ }^{5}$.

Es Didáctica de las Ciencias Sociales una materia de 9 créditos de carácter obligatorio que se imparte en la Universidad de Granada durante el segundo año del Grado en Educación Primaria de forma anual junto con otras tales como: Didáctica de la Lengua española II (9 créditos), Dificultades de aprendizaje (6), Recursos didácticos y tecnológicos (6), Enseñanza y aprendizaje de las matemáticas (6), Idioma extranjero y su didáctica (6), Atención a la diversidad (6), Enseñanza de la Educación Física (6) y Organización de centros educativos (6).

Su contenido incluye cuestiones de Epistemología de las CCSS así como elementos de Geografía, Historia, Historia del Arte, Sociología y Antropología, junto a su correspondiente tratamiento didáctico, tales como: el espacio geográfico, el tiempo histórico; las personas, las culturas, las instituciones y la organización social a lo largo del tiempo; educación histórica y formación para la ciudadanía y los derechos democráticos; y la actividad humana en el medio. Asimismo integra otros elementos técnicos relativos a la inserción de las CCSS en el currículo de Educación Primaria, la programación por competencias de contenidos de CCSS o el análisis de recursos concretos como los itinerarios didácticos o los trabajos de campo.

Las competencias que se busca alcanzar mediante su desarrollo son de tipo general (Instrumentales, personales, sistémicas, disciplinares y profesionales) y específico, figurando entre estas últimas las siguientes: Comprender los principios básicos de las CCSS; conocer el currículo escolar de las CCSS; integrar el estudio histórico y geográfico desde una orientación instructiva y cultural; fomentar la educación democrática de la ciudadanía y la práctica del pensamiento social crítico; valorar la relevancia de las instituciones públicas y privadas para la convivencia pacífica entre los pueblos; conocer el hecho religioso

\footnotetext{
${ }^{5}$ Véase la Memoria del título de Grado en Maestro en Educación Primaria. Universidad de Granada (2010: 21-30), verificada positivamente por la ANECA/AGAE, en http://vicengp.ugr.es/pages/_grados-verificados/43maestroeducacionprimariaverificado, pág. 59 [consultado el 25.10.2011] junto con la Guía docente de la materia elaborada por el Departamento de Didáctica de las CCSS disponible tanto en la Web de este Departamento como en la de la propia Facultad de Ciencias de la Educación de la Universidad de Granada http://fcce.ugr.es/ [consultado el 25.10.2012]
} 
a lo largo de la historia y su relación con la cultura; y desarrollar y evaluar contenidos del currículo mediante recursos didácticos apropiados promoviendo las correspondientes competencias en los estudiantes.

Los objetivos perseguidos mediante la incorporación de esta materia al currículo del Grado de Maestro en Educación Primara, recogidos en su Guía Didáctica como "resultados esperables de su enseñanza", ascienden a trece:

1. Conocer y comprender los principios básicos de las Ciencias Sociales.

2. Conocer las principales disciplinas del área de CCSS, su origen y evolución, así como sus fundamentos epistemológicos e historiográficos.

3. Integrar el estudio de las CCSS desde una óptica instructivo-cultural.

4. Elaborar propuestas didácticas relacionadas con el conocimiento y la conservación del entorno social y cultural.

5. Elaborar y defender de argumentos y la resolución de problemas dentro del área de estudio de las Ciencias Sociales.

6. Conocer y utilizar el lenguaje, los conceptos, los procedimientos explicativos y los procedimientos de investigación-verificación de las CCSS como forma de aproximación crítica al mundo social y así poder valorar la relevancia de las instituciones públicas y privadas para la convivencia pacífica entre los pueblos.

7. Fomentar la educación democrática de la ciudadanía y la práctica del pensamiento social crítico.

8. Fomentar la defensa de los derechos humanos desarrollando competencias en la comprensión de la sociedad sin discriminaciones por razón de sexo, cultura, religión, etc.

9. Diseñar, planificar y evaluar procesos de enseñanza y aprendizaje, tanto individualmente como en colaboración con otros docentes.

10. Conocer y aplicar metodologías y técnicas básicas de investigación educativa en la enseñanza de las CCSS; diseñar proyectos de innovación identificando indicadores de evaluación.

11. Revisar en el pensamiento del alumnado los materiales curriculares y los medios de comunicación, las principales estrategias de distorsión que proporcionan una visión simple en exceso y con prejuicios del mundo social.

12. Analizar el hecho religioso en la $\mathrm{H}^{\mathrm{a}}$ y su relación con la cultura.

13. Poseer y comprender conocimientos en las áreas de Geografía, $H^{a}$ y Arte.

El temario se concreta en nueve prácticas desarrolladas en Seminarios y Talleres y en los siguientes siete bloques temáticos: Epistemología de las CCSS; El espacio geográfico y su tratamiento didáctico; El tiempo histórico y su tratamiento didáctico; Aproximación a otras Ciencias Sociales; Las CC.SS. en el currículum de Educación Primaria. El área de Conocimiento del Medio Social y Cultural; Educación histórica y formación para la ciudadanía desde las Ciencias Sociales; Propuestas de aprendizaje para las Ciencias Sociales. Bloques de contenido que se refuerzan y apoyan mediante una selección bibliográfica básica. 
La metodología establecida para la materia de Didáctica de las CCSS se concreta en lecciones magistrales, actividades prácticas, actividades no presenciales individuales, actividades no presenciales grupales y tutorías. La evaluación se efectúa mediante los siguientes instrumentos: Asistencia, participación y aprovechamiento en clase; confección de trabajos individuales y en grupo sobre la temática trabajada y sugerida durante el desarrollo de los diferentes temas; elaboración de informes y pruebas escritas; realización de visitas y/o itinerarios programados, reales o virtuales, conforme a criterios y guías elaborados o sugeridos en clase. Los criterios de calificación de las producciones realizadas serán: calidad y profundidad de las ideas propias o, en su caso, del grupo; calidad, profundidad y coherencia del contenido manejado y de los argumentos utilizados; claridad y presentación adecuadas. Sobre el sistema de calificaciones adoptará una calificación numérica, según indica el artículo 5 del Real Decreto 1125/2003, de 5 de septiembre de 2003, por el que se establece el crédito europeo y el sistema de calificaciones en las titulaciones universitarias de carácter oficial así como su validez de en el territorio nacional.

\section{Algunas claves de nuestra propuesta.}

Tras haber caracterizado las principales coordenadas y el contexto en que tiene lugar la formación inicial del profesorado de Educación Primaria, intentar definir los retos formativos planteados a los responsables de esta tarea y realizada una primera valoración de la contribución de la Didáctica de las CCSS como área de conocimiento a la formación del profesorado de Educación Primaria, pasaremos a enumerar a continuación algunas cuestiones que desde nuestro punto de vista deben ser tenidas en cuenta a la hora apreciar en su justa medida las implicaciones establecidas entre tal área de conocimiento y el proceso de formación inicial del profesorado.

$1^{\circ}$. Encontramos que la primera relación que debe establecerse entre ambas realidades se sitúa en el nivel de la reflexión teórica; constatación que efectuamos desde la convicción de que toda actividad (investigadora y docente) propia del área de Didáctica de las CCSS debe hallarse actualizada respecto al estado de la cuestión y al conocimiento de la literatura especializada propia pues, de lo contrario, su eficacia puede verse disminuida al desconocer el docente la situación de su particular ámbito de conocimiento. Así lo hemos comprobado en nuestro quehacer cotidiano y por ello entendemos que toda acción profesional de calidad debe encontrarse al día en relación a los problemas surgidos respecto a la definición del conocimiento didáctico elaborado para la enseñanza universitaria de las CCSS, sobre todo, si se busca iniciar al alumnado en un proceso personal de autoformación basado en la reflexión efectuada desde la práctica docente.

$2^{\circ}$. En continuidad con lo anterior juzgamos también necesario que todo debate académico quede enlazado con al resto de discusiones epistemológicas y sociales planteadas en el Área por pura coherencia con las correspondencias establecidas en el trinomio sociedad-ciencia-educación; aspecto este sobre el que, desde nuestra experiencia, encontramos pertinente que el alumnado reconozca la relevancia de la enseñanza de los contenidos sociales con el fin de llevarle al descubrimiento de la necesidad de que las grandes cuestiones de interés social hallen eco en la escuela. Asimismo resulta esencial que los estudiantes aprendan a reflexionar sobre el sentido educativo de la enseñanza de las CCSS y las diversas respuestas barajadas al respecto en las diferentes corrientes epistemológicas. 
$3^{\circ}$. Dando un paso más, parece evidente que la Didáctica de las CCSS contribuye igualmente, desde la especificidad disciplinar de sus objetivos, contenidos y finalidades, al desarrollo del rico entramado de competencias asumidas en el currículo formativo del profesorado de Educación Primaria, especialmente en razón de su vocación social y humanística. Por todo ello cabría afirmar que el horizonte competencial hacia el que los docentes en formación se encaminan -muy indicativo de las implicaciones establecidas entre las CCSS y la formación inicial de los maestrosdebería responder a las siguientes exigencias:

$\checkmark$ Dominar los contenidos disciplinares y didácticos de las diversas CCSS así como la relación interdisciplinar entre las mismas.

$\checkmark$ Diseñar, planificar y evaluar procesos de enseñanza-aprendizaje dotados de contenidos sociales; plantear y regular espacios de aprendizaje en contextos de diversidad que atiendan tanto cuestiones de sentido como aspectos relativos a la igualdad de género, la equidad y el respeto o los derechos humanos.

$\checkmark$ Colaborar con todos los sectores de la comunidad educativa y su entorno asumiendo la dimensión educadora de la función docente, fomentando la educación democrática y desarrollando procesos de formación integral.

$\checkmark$ Mantener una relación crítica y autónoma respecto a saberes, valores e instituciones sociales públicas y privadas para poder reflexionar sobre las prácticas de aula con el fin de introducir líneas de innovación y mejora de la docencia.

$\checkmark$ Asumir que el ejercicio de la función docente debe perfeccionarse y adaptarse con el tiempo a los cambios científicos, pedagógicos y sociales adquiriendo a la vez hábitos y destrezas para el aprendizaje autónomo y cooperativo.

$\checkmark$ Conocer y aplicar las tecnologías de la información y de la comunicación y aprender a discernir de modo selectivo la información audiovisual que pueda contribuir a los aprendizajes, a la formación cívica y a la riqueza cultural.

$\checkmark$ Comprender la función, posibilidades y límites de la educación en la sociedad actual junto con las competencias fundamentales que afectan a los centros educativos.

Tal conjunto de exigencias, entendidas como expresión de las implicaciones establecidas entre la formación del profesorado y la Didáctica de las CSS, pensamos que garantiza de forma adecuada la incorporación e implementación de los objetivos generales del Grado en Maestro de Educación Primaria, las competencias generales del mismo, las propias de la titulación y las competencias específicas asignadas en dicho Grado al módulo de Enseñanza y aprendizaje de las Ciencias Sociales, asi como el desarrollo de otras capacidades actitudinales ${ }^{6}$ y un elemento tan ineludible en cualquier proyecto formativo de docentes como es el componente social y de educación en valores. De forma ilustrativa podríamos recordar que, en el caso de España, su actual ordenamiento jurídico (LOE, R.D. de contenidos mínimos 1513/2006, Anexo I) establece como meta a alcanzar la capacitación del futuro docente para que, con la ayuda de la Didáctica de las CCSS, adquiera las necesarias capacidades en orden al desarrollo de ocho grandes competencias establecidas para los escolares de Ed. Primaria?. Conjunto competencial

\footnotetext{
${ }^{6}$ En este campo la implicación de la Didáctica de las CCSS en la formación del profesorado debería garantizar al menos tres cuestiones: el desarrollo del sentido ético y valorativo de los actos educativos y de enseñanza de las CCSS, la potenciación de la empatía y el respeto a la diversidad y, finalmente, la demostración de actitudes cívicas junto al aprecio de la importancia de educar para el ejercicio de la ciudadanía.

${ }^{7}$ 1. Competencia en comunicación lingüistica; 2 Competencia matemática; 3 Competencia en el conocimiento y la interacción con el mundo físico; 4 Competencia en el tratamiento de la información y competencia digital; 5 Competencia social y ciudadana; 6 Competencia cultural y artística; 7 Competencia para aprender a aprender; y 8 Competencia para la autonomía e iniciativa personal.
} 
del que, por coherencia disciplinar, constituyen para nosotros objeto de particular interés las competencias número cinco, Social y Ciudadana, y seis, Cultural y Artisticas.

$4^{\circ}$. Para completar el análisis de tal proceso, debe reseñarse que la contribución de las CCSS a la formación del profesorado se muestra también en el hecho de que el conjunto de objetivos principales de la materia tenga por objeto fundamentar crítica y razonadamente la enseñanza de las CCSS orientando así su futura práctica docente. Para ello resulta esencial desarrollar adecuadamente los conocimientos, recursos y habilidades que favorezcan la toma de decisiones profesionales. Asimismo, la adquisición de elementos de análisis para interpretar, recrear y diseñar la práctica pedagógica debe hacerse desde el análisis del currículum y sus componentes didácticos.

$5^{\circ}$. Otra posibilidad derivada de la mutua implicación entre las CCSS y la formación del profesorado, no siempre tenida en cuenta, consiste en el hecho de que el alumnado asuma tal disciplina como parte esencial de su modelo docente en el futuro desempeño profesional. Consideramos que es esta una realidad en que deben tener parte activa los principios constructivistas del aprendizaje significativo. No obstante desearíamos dejar claro al mismo tiempo que en modo alguno deben rechazarse totalmente posibilidades más tradicionales que contemplan tanto clases expositivas como otras de carácter práctico y participación activa.

$6^{\circ}$. En continuidad con lo anterior entendemos que desde la disciplina de la Didáctica de las CCSS se ofrece además la posibilidad de que los profesores en formación tomen conciencia de la dimensión política y ética de la enseñanza mediante actividades de investigación, presupuestos normativos y el repertorio de valores subyacente a todo modelo teórico junto con sus propuestas de acción. Conjunto este que se completa con la posibilidad de analizar y potenciar los valores personales de cada alumno; su consistencia y articulación unida a la toma de conciencia moral y a las opciones valorativas que posee cada alumno respecto a las prácticas educativas y a su futuro profesional como fundamentos de su futura dimensión de "profesor reflexivo".

$7^{\circ}$. Junto a tales parámetros de referencia, entendemos que la Didáctica de las CCSS favorece de igual modo la formación del profesorado al tratarse de un instrumento conceptual y metodológico de iniciación del futuro docente muy eficaz para abordar el análisis de la realidad de los procesos de enseñanza-aprendizaje y su proyección en coherencia con sus propios compromisos morales. Al respecto hemos comprobado cómo tales procesos facilitan al estudiante la elaboración, desde el conocimiento teórico y práctico de la materia, de esquemas conceptuales válidos para efectuar interpretaciones críticas y cuestionar sus creencias mediante un proceso propiciador de su práctica educativa. Igualmente se ha constatado la eficacia derivada de ligar el contenido con la realidad social y educativa del entorno próximo y los conocimientos previos propios; vía por la cual es posible incorporar la pluralidad de ideas existente en orden a la elaboración de un análisis de lo social, en función de la interpretación ofrecida por las diferentes corrientes teóricas.

$8^{\circ}$. Consideramos también que la capacidad para interpretar situaciones reales de enseñanza/aprendizaje en función de contextos sociales y escolares, la habilidad en la gestión de

\footnotetext{
8 Junto a las competencias, otro doble referente para establecer el marco de toda acción docente en este ámbito geográfico son los objetivos generales del Grado en Maestro de Educación Primaria y las competencias generales y específicas de dicha titulación ya aludidas. Véase el R. D. 55/2005 de 21 de enero (Boja n 21 de 25/01/2005) y la Memoria del titulo de Grado en Maestro en Educación Primaria. Universidad de Granada (2010: 21-30), verificada positivamente por la ANECA/AGAE, en http://vicengp.ugr.es/pages/ gradosverificados/43maestroeducacionprimariaverificado [consultado el 21.10.2011]
} 
la vida del aula, la lucidez para adoptar decisiones fundamentadas y la posibilidad de valorar las consecuencias de las mismas, forman asimismo parte de las actividades docentes que deben ser potenciadas desde la Didáctica de las CCSS. El aprendizaje de todas ellas durante la formación inicial es una posibilidad que ofrece la oportunidad de cuestionar y reformular tanto las concepciones previas como las observadas en las prácticas para proyectar acciones futuras.

$9^{\circ}$. Para concluir, hemos de advertir sobre la existencia de un grave obstáculo que distorsiona significantemente la contribución de la Didáctica de las CCSS a la formación del profesorado: la constatación de cómo las anteriores acciones deben abordarse teniendo en cuenta que la actual generación de estudiantes ofrece, en líneas generales, un deficiente nivel académico y cultural tal como han confirmado los últimos informes PISA en España y los resultados de ENLACE en México9. Sin entrar a valorar sus causas, ello representa una gran dificultad para lograr la implementación de procesos de incentivación de sus inquietudes intelectuales, sobre todo, cuando afloran limitaciones de compresión o actitudes de rechazo ante corrientes renovadoras en el ámbito del conocimiento didáctico. Por tal razón juzgamos que en estos momentos toda labor docente ha de plantearse como un proceso paulatino de desarrollo competencial que ofrezca a los estudiantes la oportunidad de asumir el reto de descubrir nuevas inquietudes y experimentar cómo sus dudas les impulsan a la búsqueda de respuestas iniciándose asi en la aventura del saber de forma independiente.

\section{Referencias bibliográficas}

AA.VV. (2003): La globalización de los derechos humanos. Crítica: Barcelona.

ACOSTA, J. (2001): "Ciudadanía, cosmopolitismo y nacionalismo", En J. DE PRADO (coord.): Diversidad cultural, identidad y ciudadanía. INET: Córdoba, págs. 215-236.

ARANGUREN, C. (1999): "La condición unitaria de lo valorativo/cognitivo en la enseñanza y aprendizaje de las Ciencias Sociales", En Teoría y Didáctica de las Ciencias Sociales, 4 (1999) págs. 195-205.

AVERY, P. G. (2004): "Social studies teacher education in an era of globalization". En ADLER, S.; Critical issues in social studies teacher education. Greenwich: Conn, págs. 37-57.

BIFANI, P. (2002): La globalización: ¿Otra caja de Pandora? Granada: Universidad de Granada.

CARBONELL I PARISS, J. (2005): Educar en tiempos de incertidumbre. Equidad e interculturalidad en la escuela. Madrid: Ministerio de Educación y Ciencia.

CARBONELL, F. (2008): "Retos y dificultades en la implementación en las aulas de las políticas de acogida e integración", En Experiencias de acogida e integración educativa de alumnado inmígrate iberoamericano. Madrid: Ministerio de trabajo-Organización de Estados Iberoamericanos, págs. 7-20.

CARNOY, M. (1999): Globalization and educational reform: what planners need to know. Paris: International Institute for Educational Planning.

CARR, D. (2005): El sentido de la educación. Una introducción a la filosofía y a la teoría de la educación y de la enseñanza. Graó: Barcelona.

CASALS, J. (2006): "Se aprueba la LOE y empezará a aplicarse en el próximo curso" En Cuadernos de Pedagogía, 358 (2006) págs. 12-15.

CASTELLS, M. (1997): La era de la información. Economía, sociedad y cultura. Vol. 2. El poder de la identidad. Alianza Editorial: Madrid.

\footnotetext{
${ }^{9}$ Para profundizar en la valoración de los resultados de la prueba ENLACE en México en lo relativo al área de conocimiento de Historia véase el reciente trabajo de $\mathrm{M}^{\mathrm{a}} \mathrm{C}$. Miranda y A. Palma (2001). 
CASTELLS, M. (2001): La era de la información. Economía, sociedad y cultura. Vol. 3. El fin del milenio. Alianza Editorial: Madrid.

DELORS, J. (1996); La educación encierra un tesoro. Informe de la UNESCO de la Comisión Internacional sobre la educación para el S. XXI. Madrid: Santillana/UNESCO.

DRUCKER, P. F. (1993): Post-Capitalist Society. Harper Business: New York.

DRUCKER, P. F. (1994): "The Age of Social Transformation" En The Atlantic Monthly, Vol. 273n n 11 (1994), Boston. http://www.theatlantic.com/election/connection/ecbig/soctrans.htm [consultado el 04.11.2011]

ESTEVE, J. M. (2003): La tercera revolución educativa. La educación en la sociedad del conocimiento. Paidós: Barcelona.

FELDFEBLER, M. y VERGER, A. (2006): "Los mecanismos de mercado en la educación. Una aproximación a sus efectos sociales". En Cuadernos de Pedagogía, 353 (2006) págs. 60-63.

FERNÁNDEZ ENGUITA, M. (2001): Educar en tiempos inciertos. Morata: Madrid.

GIMENO, J. (2005): La educaciōn que aún es posible. Morata: Madrid.

HARGREAVES, A. (2003): Enseñar en la sociedad del conocimiento. Octaedro: Barcelona.

KUBOW, P.; GROSSMAN, D.; NINOMIYA, A. (2000) Multidimensional citizenship:Educational policy for the 21st century, in Cogan, J. J. (ed), Citizenship for the 21st century: An international perspective on education. Kogan Page: London

LEY ORGANICA 2/2006 DE EDUCACION, LOE, BOE nº 106 de 4.10. 2006. Madrid.

LICERAS, A. (1993): Didáctica de las Ciencias Sociales en Educación Primaria. Sevilla: Algaida.

LUENGO, J. J. (2005) (comp.): Paradigmas de gobernación y de exclusión social en la educación. Fundamentos para el análisis de la discriminación escolar contemporánea. Pomares: Barcelona.

MIRALLES, R. (2005): "Repensar la reforma, reformar el pensamiento. Edgar Morin". En Cuadernos de Pedagogía, 342 (2005) págs. 42-46.

MIRANDA, Ma C. y PALMA, A. (2011): "Evaluación de los resultados de aprendizaje del Área de Historia del Currículo Oficial Mexicano en el nivel de Enseñanza Secundaria y su incidencia formativa en el alumnado" en P. MIRALLES; S. MOLINA, S.; A. SANTISTEBAN (eds.): La evaluación en el proceso de enseñanza y aprendizaje de las Ciencias Sociales. Vol. I. Murcia: Asociación de profesores universitarios de Didáctica de las CCSS, págs. 269-276.

MORIN, E. (2004): Los siete saberes necesarios para la educación del futuro. Paidós: Barcelona.

MULET, B. (2003) : Globalització I ciéncies socials. Limits I dilemes. Mallorca: Universitat de les Illes Balears.

MUSSBAUM, M. C. (1999): Los límites del patriotismo. Identidad, pertenencia y ciudadanía mundial. Paidós: Barcelona.

NIETO, J. M. (2001): La autoevaluación del profesor: cómo puede el profesor evaluar y mejorar su práctica docente. Barcelona: Ciss Praxis.

PÉREZ, A.; SOLA, M.; BLANCO, N. y BARQUİN, J. (2004): "Luces y sombras en la situación profesional de los docentes españoles" En GIMENO, J. y CARBONELL, J. (coords.): El sistema educativo. Una mirada crítica. Praxis: Barcelona, págs. 127-141.

RAMBLA, X. y BONAL, X. (2006): "La desigualdad que no cesa" En Cuadernos de Pedagogía, 353 (2006) págs. 50-54.

RAMBLA, X.; VALIENTE, O. y CONSTANS, M. (2006): "Tendencia actual en Europa y América Latina" En Cuadernos de Pedagogía, 353 (2006) págs. 55-59.

TEDESCO, J. C. (1995): El nuevo pacło educativo. Educación, competitividad y ciudadanía en la sociedad moderna. Anaya: Madrid.

TORRES, J. (1998): Globalización e interdisciplinariedad: el currículum integrado. Morata: Madrid.

TOURAINE, A. (2005): Un nuevo paradigma para comprender el mundo de hoy. Paidós: Barcelona.

WHITSON, J. A. (2004): "What social studies teachers need to know. The new urgency of some old disputes" En S. ADLER: Critical issues in social studies teacher education. Greenwich: Conn, págs. 9-35. 